\title{
Avaliação da resistência de união de restaurações de resina composta com diferentes sistemas adesivos, em dois tempos de armazenamento
}

Composite resin bond strength with different dentin-bonding agents in two storage times

Fernando Zurlo Dellazzana1, Fábio Herrmann Coelho-De-Souza², Celso Afonso Klein-Júnior³,

${ }^{1}$ Cirurgião-Dentista formado pela ULBRA de Cachoeira do Sul

\section{Resumo}

A degradação hidrolítica dos adesivos dentinários pode influenciar a resistência de união das restaurações de resina composta ao longo do tempo. O objetivo deste trabalho é avaliar a influência do tempo de armazenamento associada à termociclagem e do tipo de adesivo dentinário empregado sobre a resistência de união de restaurações de resina composta. Foram utilizados 20 molares hígidos, os quais foram divididos em 2 tempos de avaliação ( 24 horas e 6 meses + termociclagem). Cada dente teve a dentina relativa à superfície oclusal exposta, a qual recebeu 3 corpos de prova de resina Flow com os sistemas adesivos: Scotchbond multiuso (3M/ESPE - Grupo 1), ClearFil SE Bond (Kuraray - Grupo 2) e Adper Single Bond (3M/ESPE - Grupo 3). Após o teste de microcrocisalhamento, os dados foram analisados estatisticamente pelos testes ANOVA, Tukey e t-student $(\alpha=0,05)$. Os resultados mostraram que os adesivos Scotchbond multiuso, ClearFil SE Bond e Single Bond não apresentaram diferenças na resistência de união em 24 horas de armazenamento. Após 6 meses, o adesivo ClearFil SE Bond obteve resistência superior ao Scotchbond multiuso. $\mathrm{O}$ adesivo Scotchbond multiuso sofreu redução na resistência de união após 6 meses de armazenamento. Os adesivos ClearFil SE Bond e Single Bond não sofreram influência do tempo de armazenamento e da termociclagem.

Palavras-chave: Adesivos dentinários, Resinas compostas, Resistência de união

\begin{abstract}
Water degradation of dentin bonding agents may influence bond strength in composite resin restorations after some periods of time. The aim of this study was to evaluate the influence of storage time with thermociclyng and type of dentin bonding agents used on the composite resin restorations microshear bond strength. Twenty molars were used and divided into two evaluation periods (24 hours and 6 months, plus thermocycling). Each tooth had its occlusal surface dentin exposed, and received three units of test made of Flow composite resin, with the following adhesive systems: Scotchbond multipurpose (3M/ESPE - group 1), Clearfil SE Bond (Kuraray group 2) and Adper Single Bond (3M/ESPE - group 3). Subsequently to the microshear bond strength testing, data were analyzed by ANOVA, Tukey and Student $t$ tests $(\alpha=0,05)$. The results showed that there were no differences between Scotchbond multipurpose, ClearFil SE Bond and Adper Single Bond on microshear bond strength after 24 hours of storage. After 6 months, ClearFil SE Bond demonstrated higher resistance than Scotchbond multipurpose. Scothtchbond multipurpose presented a reduction in its performance when 6 months passed. ClearFil SE Bond and Adper Single Bond suffered no influence resultant from the storage period.
\end{abstract}

Keywords: Dentin-bonding agents, Bond strength, Composite resins
${ }^{2}$ Doutor em Dentística pela UFPel e Professor de Dentística da UFRGS

${ }^{3}$ Doutor em Ciência dos Materiais pela UFRGS; Professor de Técnicas e Materiais em Dentística e Clínica Integral na ULBRA de Cachoeira do Sul

Correspondência: Prof. Fábio Herrmann Coelho-de-Souza

Endereço: Rua Ramiro Barcelos, 2492 - CEP 90035-003 - Bairro Santana

Porto Alegre - RS

E-mail: fabio.herrmann@yahoo.com.br

\section{Introdução}

A Odontologia adesiva está cada vez mais presente na prática restauradora, a adesão ao esmalte e à dentina passou a ser uma rotina e uma realidade na Dentística atual. No que se refere ao substrato dental, obteve-se uma união satisfatória, devido ao desenvolvimento de inúmeros sistemas adesivos. Enquanto a adesão em esmalte é considerada uma técnica segura e confiável, a união à dentina ainda é um desafio, por ser um substrato heterogêneo, com estrutura canalicular, alto conteúdo orgânico e intrinsecamente úmido (REIS et al., 2001). A busca pelo material ideal em termos de adesão à estrutura dentinária tem demonstrado, então, um desafio a ser superado.

As primeiras tentativas de união das resinas ao esmalte foram feitas em 1955, por Buonocore, e continuam sendo largamente pesquisadas, a fim de reproduzir, com grande fidelidade, a estrutura dentária perdida e um melhor vedamento marginal. Com as técnicas de condicionamento ácido total, esmalte e dentina (FUSAYAMA et al., 1979), o condicionamento ácido promove microporosidades no esmalte, e na dentina, com auxílio dos primers, vai ocorrer um entrelaçamento do adesivo com as fibras colágenas, formando a camada híbrida (NAKABAYASHI; KOJIMA; MASUHARA, 1982; VAN MEERBEEK et al., 2003). Ou ainda, com o emprego dos adesivos autocondicionantes, os primers acidificados (Phenyl-P e Hema) fazem a modificação e interação com o smear layer e promovem a integração com o substrato (PERDIGÃO; RITTER, 2001).

Apesar da evolução dos sistemas adesivos atuais, a capacidade de adesão dos materiais restauradores à estrutura dentária ainda tem sido alvo de diversas pesquisas (DE MUNCK et al., 2005). A cavidade oral é um desafio para a dinâmica adesiva, tendo em vista a constituição da microbiota bucal e $\mathrm{pH}$, atividade enzimática e presença de umidade, fazendo com que as restaurações não se comportem de forma estável ao longo do tempo (SANO, 2006). O simples armazenamento das restaurações de resina composta em água pode fazer com que seja reduzida a sua resistência de união (KATO; NAKABAYASHI, 1998; SADECK, TAVARES; CARDOSO, 2003; COELHO-DE-SOUZA et al., 2008).

A fragilização das restaurações de resina composta durante o tempo de armazenamento pode ser associada à presença de água dentro da camada híbrida, ocorrendo o que se denomina nanoinfiltração (SANO et al., 1995), à degradação hidrolítica do adesivo (LELOUP et al., 2001) ou à degradação das fibras colágenas desmineralizadas e não impregnadas pelo adesivo (PASHLEY et al., 2004). Dessa forma, a durabilidade das restaurações adesivas mostra-se influenciada por diversos fatores, inclusive pelo tipo de sistema adesivo empregado (TAVARES; CONCEIÇÃO, 2004) e necessita maiores investigações.

Assim sendo, o objetivo do presente trabalho foi avaliar a resistência de união em dentina, através do ensaio de microcisaIhamento, de restaurações de resina composta com três sistemas 
de união (Scotchbond multiuso/3M-ESPE; Adper Single Bond/3MESPE; e ClearFil SE Bond/Kuraray), avaliados em 2 tempos de armazenamento: 24 horas e 6 meses associado à termociclagem.

\section{Materiais e Métodos}

Para a realização do presente trabalho, foram utilizados 20 dentes humanos, molares hígidos, extraídos por razões terapêuticas (CEP - ULBRA 2006 - 096H), submetidos a uma profilaxia prévia (pedra-pomes + água + escova Robson) e armazenados em solução de Cloramina $0,5 \%$ por uma semana para desinfecção (ISO 11405). Os dentes foram divididos em 3 grupos (Quadro 1). No Grupo 1, foi utilizado sistema adesivo de 3 passos (Scotchbond multiuso / 3M ESPE); no Grupo 2, sistema adesivo autocondicionante (ClearFil SE Bond / Kuraray) e no Grupo 3 foi utilizado sistema adesivo de 2 passos (Adper Single Bond / 3M ESPE). Esses Grupos foram, então, subdivididos em $A$ e $B$, sendo que em $A$ os dentes ficaram armazenados 24 horas em água $(n=10)$ e em $B$ os dentes foram armazenados 6 meses em água à temperatura ambiente $(n=10)$, associados à termociclagem.

Quadro 1. Grupos experimentais.

\begin{tabular}{lll}
\hline \multicolumn{1}{c}{ GRUPOS } & TEMPO A & TEMPO B \\
\hline 1- Scotchbond multiuso & 24 horas & 180 dias \\
2- ClearFil Se Bond & 24 horas & 180 dias \\
3- Adper Single Bond & 24 horas & 180 dias \\
\hline
\end{tabular}

SBMU - Scotchbond multiuso, CF - ClearFil SE Bond, SB - Adper Single Bond.

Para que a superfície dentinária dos molares fosse exposta, foi seccionada a face oclusal com o auxílio de um disco diamantado dupla face (KG Sorensen) em baixa rotação, sob refrigeração com água corrente até que toda a superfície de dentina fosse exposta (cavidade de profundidade média). A seguir, os dentes foram incluídos individualmente, em resina acrílica autopolimerizável (JET/ Clássica), em um cilindro plástico (anéis de PVC) de $25 \mathrm{~mm}$ de diâmetro por $20 \mathrm{~mm}$ de altura, de tal maneira que a superfície oclusal preparada em dentina ficasse voltada para a superfície externa e superior do anel. Após, o conjunto PVC/resina acrílica/dente foi submetido a uma sequência de lixas, com o auxílio de lixas d'água de granulações \#300 e \#600 na superfície superior. Com a finalidade de delimitar a área de aplicação dos sistemas adesivos, foi empregada uma película adesiva transparente (máscaras de papel Contact), em que foram confeccionadas três perfurações centrais com o maior orifício da plataforma do alicate perfurador de Ainsworth. Com isso, foi delimitada a área a ser efetuada a união dentina/adesivo/material restaurador. Logo após, foram confeccionados corpos de prova com resina composta fotoativada de baixa viscosidade (Natural Flow DFL) na cor $A 3$, tendo, então, um diâmetro de $1,2 \mathrm{~mm}^{2}$ para cada corpo de prova (figura 1). Foi calculada a área de união, através da formúla $A=\pi r^{2}$, onde $A=3,14 \times(0,6)^{2}$, e a área final foi de $A=1,13$. Os corpos de prova foram confeccionados com auxílio de uma matriz de silicone de $1,2 \mathrm{~mm}^{2}$ de diâmetro e $2 \mathrm{~mm}$ de altura. $\mathrm{O}$ aparelho fotopolimerizador (Gnatus) empregado foi do tipo convencional à luz halógena, com intensidade de luz medida em $500 \mathrm{~mW} / \mathrm{cm}^{2}$, calibrado em radiômetro Demetron.

Após a adaptação das máscaras (papel Contact) na superfície de dentina exposta, essa foi condicionada com ácido fosfórico a $37 \%$ (ATACK TEC) por 15 segundos para o Grupo 1, seguido de lavagem por 15 segundos e secagem estratégica com papel absorvente, mantendo a superfície levemente úmida e, a seguir, o sistema adesivo Scotchbond multiuso foi aplicado: o primer foi aplicado utilizando-se um aplicador descartável microbrush (KG brush ( KG SORENSEN) na superfície de dentina exposta e, depois, um jato de ar bem superficial para remover o excesso de solvente; em seguida, foi aplicado o adesivo, utilizando-se outro microbrush, e foi fotopolimerizado por 20 segundos. No Grupo 2, o sistema adesivo autocondicionante de dois frascos (ClearFil SE Bond) foi aplicado de acordo com o fabricante: antes de aplicar o primero, foi secada a superfície, e, então, primeiramente se aplicou o primer sobre a dentina, após secagem suave com jato de ar; a seguir, aplicação do adesivo e, em seguida fotoativação por 10 segundos. No Grupo 3, com sistema adesivo de 2 passos, após a secagem com jato de ar de toda a dentina exposta, essa foi condicionada com ácido fosfórico a $37 \%$ por 15 segundos, seguido de lavagem por 15 segundos e secagem estratégica com papel absorvente, mantendo a superfície de dentina levemente úmida; a seguir, o sistema adesivo Adper Single Bond foi aplicado, utilizando um aplicador descartável microbrush com 2 cobrimentos consecutivos, intercalados por jato de ar e, em seguida, foi fotopolimerizado por 10 segundos. Após aplicação de cada um dos Sistemas Adesivos, a matriz de silicone foi posicionada para ser inserida a resina composta Flow em incremento único, fotoativada por 20 segundos, com aparelho fotoativador justaposto à matriz.

Os corpos de prova do Grupo A foram armazenados em água por 24 horas, para, então, serem levados até a máquina de ensaio universal Versat 500 (Pantec), utilizando-se uma célula de carga de 250 Newtons, com uma velocidade de $0,5 \mathrm{~mm} / \mathrm{min}$, sendo a linha de união perpendicular ao solo; a resina foi apreendida com fio de amarria Sutupack Aciflex - aço monofilamentado 2-0 (Ethicon Johmson \& Johmson), fazendo uma ancoragem da peça junto à linha de união. Então, com movimento de tração, a máquina induziu ao microcisalhamento (Figura 2). Os valores da máquina foram fornecidos em Newtons e foram divididos pela área de união, para obterem-se os valores em megapascal (MPa). Após, foi avaliado o padrão da área fraturada no microscópio Estereoscópico com aumento de 30x, no qual foi observado o tipo de fratura ocorrido na superfície de dentina: fratura adesiva (fratura ocorrida na linha de união entre o corpo de prova e o sistema adesivo), fratura coesiva (fratura do material restaurador), fratura dentinária (deslocamento do corpo de prova e de uma porção de dentina junto) e fratura mista (associação de mais de um tipo de fratura juntas). Os corpos de prova do Grupo B foram armazenados em água por 6 meses, associados à Termociclagem, com 500 ciclos de temperatura variando de $5^{\circ} \mathrm{C}$ a $55^{\circ} \mathrm{C}$, com 30 segundos de permanência, para, então, submeterem-se aos mesmos procedimentos descritos anteriormente.

\section{Resultados}

Os dados obtidos no ensaio de microcisalhamento, no tempo de 24 horas e 6 meses, foram tabulados e submetidos à análise estatística com um nível de significância de $5 \%(\alpha=0,05)$.

Para o período de 24 horas, foi empregado o teste de análise de variância (ANOVA) (Tabela 1).

Para o período de 6 meses de armazenamento, foi empregado o teste de análise de variância (ANOVA), o qual mostrou haver diferença estatisticamente significativa entre os grupos $(p<0,05)$. Para identificar o contraste entre os grupos, foi utilizado o teste de comparações múltiplas de Tukey (Tabela 2).

Para realizar a comparação entre os períodos de 24 horas (tempo A) e 6 meses (tempo B), foi utilizado o teste t-student (Tabela 3).

Tabela 1. Comparação dos valores de resistência de união dos grupos testados no tempo A (24 horas - valores em MPa):

\begin{tabular}{lccccc}
\hline \multicolumn{1}{c}{ Grupos } & $\mathbf{n}$ & Média & DP & IC (95\%) & p \\
\hline $1 \mathrm{~A}(\mathrm{SBMU})$ & 10 & 18,57 & 5,71 & $14,47-22,26$ & 0,47 \\
$2 \mathrm{~A}(\mathrm{CF})$ & 10 & 16,54 & 7,52 & $11,15-21,92$ & \\
$3 \mathrm{~A}(\mathrm{SB})$ & 10 & 20,07 & 5,74 & $15,96-24,17$ & \\
\hline
\end{tabular}

SBMU - Scotchbond multiuso, CF - ClearFil SE Bond, SB - Adper Single Bond, DP - Desvio-padrão, IC - Intervalo de Confiança. 
A partir da análise dos resultados anteriores, verifica-se que não houve diferença estatisticamente significativa entre os grupos testados, no período de 24 horas.

Tabela 2. Comparação dos valores de resistência de união dos grupos testados no tempo B (6 meses - valores em MPa).

\begin{tabular}{lccccc}
\hline \multicolumn{1}{c}{ Grupos } & $\mathbf{n}$ & Média & DP & IC (95\%) & p \\
\hline $1 \mathrm{~B}(\mathrm{SBMU})$ & 10 & $10,95^{\mathrm{A}}$ & 3,53 & $8,42-13,48$ & 0,038 \\
$2 \mathrm{~B}(\mathrm{CF})$ & 10 & $20,53^{\mathrm{B}}$ & 8,15 & $14,49-26,16$ & \\
$3 \mathrm{~B}(\mathrm{SB})$ & 10 & $15,15^{\mathrm{AB}}$ & 9,94 & $8,04-22,27$ & \\
\hline
\end{tabular}

SBMU - Scotchbond multiuso, CF - ClearFil SE Bond, SB - Adper Single Bond, DP - Desvio-padrão, IC - Intervalo de Confiança.

* Letras iguais não diferem entre si.

Para o período de 6 meses de armazenamento associado à termociclagem, o Grupo 2 (ClearFil SE Bond) apresentou resistência de união superior estatisticamente ao Grupo 1 (Scotchbond multiuso). O Grupo 3 (Adper Single Bond) não diferiu dos Grupos 1 e 2.

Tabela 3. Comparação dos valores de resistência de união dos grupos A e B (24 horas e 6 meses - valores em MPa).

\begin{tabular}{lccccc}
\hline \multicolumn{1}{c}{ Grupos } & $\mathbf{n}$ & Média & DP & IC (95\%) & p \\
\hline $1 \mathrm{~A}(\mathrm{SBMU})$ & 10 & 18,57 & 5,71 & $14,47-22,66$ & 0,02 \\
$1 \mathrm{~B}(\mathrm{SBMU})$ & 10 & 10,95 & 3,53 & $8,42-13,48$ & \\
$2 \mathrm{~A}(\mathrm{CF})$ & 10 & 16,54 & 7,52 & $11,15-21,92$ & 0,29 \\
$2 \mathrm{~B}(\mathrm{CF})$ & 10 & 20,53 & 8,15 & $14,49-26,16$ & \\
$3 \mathrm{~A}(\mathrm{SB})$ & 10 & 20,07 & 5,74 & $15,96-24,17$ & 0,19 \\
$3 \mathrm{~B}(\mathrm{SB})$ & 10 & 15,15 & 9,94 & $8,04-22,27$ & \\
\hline
\end{tabular}

SBMU - Scotchbond multiuso, CF - ClearFil SE Bond, SB - Adper Single Bond DP - Desvio-padrão, IC - Intervalo de Confiança.

Ao ser comparado o período de 24 horas com 6 meses, houve redução significativa da resistência de união após 6 meses de armazenamento para o Grupo 1 (Scotchbond multiuso). Para os grupos 2 e 3, o tempo de armazenamento não influenciou a resistência de união de forma significativa.

Tabela 4. Identificação dos padrões de fraturas dos grupos testados no tempo A (24 horas).

\begin{tabular}{lccccc}
\hline Grupos & Adesiva & Coesiva & Dentinária & Mista & Total \\
\hline $1 \mathrm{~A}(\mathrm{SBMU})$ & 3 & - & - & 7 & 10 \\
$2 \mathrm{~A}(\mathrm{CF})$ & 4 & - & - & 6 & 10 \\
$3 \mathrm{~A}(\mathrm{SB})$ & 2 & - & - & 8 & 10
\end{tabular}

SBMU - Scotchbond multiuso, CF - ClearFil SE Bond, SB - Adper Single Bond.

Tabela 5. Identificação dos padrões de fraturas dos grupos testados no tem po B (6 meses).

\begin{tabular}{lccccc}
\hline Grupos & Adesiva & Coesiva & Dentinária & Mista & Total \\
\hline $1 \mathrm{~B}(\mathrm{SBMU})$ & 6 & - & - & 4 & 10 \\
$2 \mathrm{~B}(\mathrm{CF})$ & 1 & - & - & 9 & 10 \\
$3 \mathrm{~B}(\mathrm{SB})$ & 7 & - & - & 3 & 10
\end{tabular}

SBMU - Scotchbond multiuso, CF - ClearFil SE Bond, SB - Adper Single Bond.

\section{Discussão}

Os resultados do presente trabalho mostraram que a resistência de união avaliada através do ensaio de microcisalhamento, da resina composta em dentina, foi igual para os três sistemas adesivos empregados no período de 24 horas; contudo, mostrou-se diferente após 6 meses de armazenamento e termociclagem. A integridade da interface adesiva das restaurações de resina composta não se comporta de forma estável ao longo do tempo (SANO, 2006). O simples armazenamento das restaurações de resina composta em água pode fazer com que seja reduzida a sua resistência de união (KATO; NAKABAYASHI, 1998; SADECK; TAVARES; CARDOSO, 2003; COELHO-DE-SOUZA et al., 2008). Kato e Nakabayashi (1998) mostraram que o período de trinta dias já foi suficiente para comprometer a resistência de união. Coelho-de-Souza e colaboradores (2008) utilizaram o tempo de 6 meses associado à termociclagem para induzir o envelhecimento de restaurações de resina composta, e mostraram que houve prejuízo na resistência à fratura e vedamento marginal.

A fragilização da interface adesiva das restaurações de resina composta, com o passar do tempo, pode ser associada à presença de água no interior da camada híbrida, ocorrendo a nanoinfiltração (SANO et al., 1995), à degradação hidrolítica do adesivo (LELOUP et al., 2001) ou à degradação das fibras colágenas desmineralizadas e não impregnadas pelo adesivo (PASHLEY et al., 2004). No presente estudo, o sistema adesivo convencional de 3 passos (Scotchbond multiuso) foi o mais afetado pelo armazenamento e termociclagem. Essa ocorrência pode estar associada ao fato de esse sistema adesivo, por apresentar passos separados de aplicação do primer e adesivo, dispor de uma maior sensibilidade técnica, devido à precisão do tempo e modo de aplicação do primer e sua secagem após a aplicação (REIS et al., 2001; VAN MEERBEEK et al., 2003). Outra situação que contribui para esse processo de redução da resistência de união é a degradação das fibras colágenas expostas pelo condicionamento que podem não ser corretamente preenchidas por esse sistema adesivo em questão, favorecendo a ação enzimática (PASHLEY et al., 2004; SANO, 2006). Em contrapartida, para os sistemas adesivos autocondicionantes, como o Clearfil SE Bond utilizado nesse estudo, essa degradação enzimática não é um fato significativo, visto que esses adesivos proporcionam uma desmineralização superficial, não havendo uma maior extensão de colágeno que dificultasse a total impregnação pelo adesivo (PERDIGÃO; RITTER, 2001; VAN MEERBEEK et al., 2003).

Wang, Shimada e Tagami (2004) avaliaram a resistência de união ao cisalhamento de dois adesivos dentinários (Adper Single Bond e ClearFil SE Bond), onde foi encontrado como resultado que a resistência dos adesivos testados diminuiu de acordo com a passagem do tempo, fato que não ocorreu no presente estudo, no qual foram utilizados os mesmo adesivos. $O$ estudo de Lodovici e colaboradores (2003), que avaliaram a influência do tempo de armazenamento ( 24 horas e três meses) na resistência adesiva de dois tipos de adesivos, um convencional (Adper Single Bond) e outro autocondicionante, mostraram que não houve diferença na resis-tência adesiva dos dois adesivos, assim como os resultados aqui apresentados. No entanto, o presente trabalho diferiu do estudo de Konno (2003), que não mostrou diferenças em 6 meses de armazenamento para restaurações com o sistema adesivo Scotchbond multiuso.

Shimada, Kikoshima e Tagami (2002) utilizaram na sua metodologia o teste de microcisalhamento, para avaliar a resistência de união de dois adesivos dentinários. Esse mesmo teste também foi empregado no presente trabalho, diferentemente dos estudos de Santos (2000), Mauro (2000) e Konno (2003) que utilizaram o teste de cisalhamento; Matos (2001) e (2004) que empregaram o teste de tração ou Sadeck, Tavares e Cardoso (2003) e Lodovici (2003) que utilizaram o teste de microtração. 
$\mathrm{Na}$ metodologia do presente estudo, foram utilizados dentes humanos molares hígidos (KANCA, 1992; MATOS et al., 2001; DANTAS et al., 2003; SHIMADA et al., 2003) e mantidos em solução de Cloramina 0,5\% por uma semana, para desinfecção (ISO 11405). Os tratamentos seguiram as recomendações de seus fabricantes quanto à técnica de aplicação dos sistemas adesivos. Os grupos foram armazenados em água por 24 horas e por 6 meses associado à termociclagem, conforme recomendação da ISO 11405, para, então, simular as diferentes trocas de temperaturas do meio bucal e a hidrólise da camada híbrida, induzindo ao enveIhecimento da linha de união (KATO; NAKABAYASHI, 1998; LODO VICl et al., 2003; KONNO et al., 2003; SANO, 2006; COELHO-DESOUZA et al., 2008).

No presente trabalho, foram observados os padrões de fraturas dos grupos testados, onde tanto para o tempo A (24 horas), quanto para o tempo $B$ (6 meses), predominaram padrões de fraturas do tipo mista (associação de mais de um tipo de fraturas juntas). Contudo, após 6 meses o número de falhas adesivas se mostrou aumentado (especialmente para os adesivos antecedidos de condicionamento ácido), o que pode estar relacionado à deterioração da camada híbrida, seja por hidrólise do adesivo (SANO, 2006), por nanoinfiltração (SANO et al., 1995; TAY; PASHLEY, 2003), por degradação do colágeno desmineralizado e não infiltrado por ação enzimática (PASHLEY et al., 2004), ou ainda pelo efeito da termociclagem sobre a interface adesiva, estressada pelas diferenças de coeficiente de expansão térmica linear entre material e substrato (ANUSAVICE, 1998; INTERNATIONAL ORGANIZATION FOR STANDARDIZATION, 2003).

O presente trabalho, dentro das suas limitações, traz uma contribuição valorosa para o conhecimento científico, uma vez que elucida a performance dos três tipos de sistemas adesivos mais empregados e indicados para restaurações de resina composta e destaca o potencial efeito do tempo de armazenamento sobre a adesão. Todavia, sugerem-se outros estudos dentro desta mesma linha de pesquisa, a fim de se verificar o desempenho dos sistemas adesivos frente a outras variáveis, como outros tempos e meios de armazenamento, fadiga mecânica, outros sistemas adesivos e, principalmente, que sejam conduzidos trabalhos de avaliação clínica para elucidar o real comportamento das restaurações em longo prazo no meio bucal.

\section{Conclusão}

A partir dos resultados do presente trabalho, segundo esta metodologia, pode-se concluir que:

- As restaurações de resina composta realizadas com os sistemas adesivos Scotchbond multiuso, ClearFil SE Bond e Adper Single Bond não se mostraram diferentes em relação à resistência de união no período de 24 horas.

- Após 6 meses de armazenamento e termociclagem, as restaurações de resina composta utilizando o sistema adesivo ClearFil SE Bond apresentaram maior resistência de união do que aquelas realizadas com o sistema adesivo Scotchbond multiuso.

- O tempo de armazenamento associado à termociclagem produziu efeito deletério na resistência de união das restaurações de resina composta que utilizaram o sistema adesivo Scotchbond multiuso, o que não ocorreu com os adesivos ClearFil SE Bond e Adper Single Bond.

\section{Referências}

ANUSAVICE, K. J. Phillips: Materiais Dentários. 10. ed. Rio de Janeiro: Guanabara Koogan, p. 412, 1998.

BUONOCORE, M. A. Simple Method of Increasing the Adhesion of Acrylic Filling Materials to Enamel Surfaces. J. Dent. Res., Washington, v.34, n.6, p. 849-853, Dec. 1955.

COELHO-DE-SOUZA, F. H. et al. Fracture Resistance and Gap Formation of MOD Restoration: Influence of Restorative Ttechnique, Bevel Preparation and Water Storage. Oper. Dent., Seattle, v. 33, n.1, p. 37-43, Jan. 2008.

DANTAS, D. C. R. E. et al. Resistência Adesiva ao Cisalhamento de Sistemas Adesivos com e sem Condicionamento Ácido Prévio. J. Bras. Clin. Odontol. Integr., Curitiba, v.7,n. 41, p. 373-376, set./out. 2003.

DE MUNCK, J. et al. A Critical Review of the Durability of Adhesion to Tooth Tissue: methods and results. J. Dent. Res., Washington, v. 84 , no. 2, p. 118-132, Feb. 2005.

FUSAYAMA, T. et al. Non-pressure Adhesion of a New Adhesive Restorative Resin. J. Dent. Res., Washington, n. 58, n.4, p. 13641370, Apr. 1979.

INTERNATIONAL ORGANIZATION FOR STANDARDIZATION. ISO 11405: Dental Materials - Testing of Adhesion to Tooth Structure. 2nd ed. Geneva, 2003.

KANCA, J. Resin Bonding to Wet Substrate. I. Bonding to Dentin. Quintessence Int., Berlin, v.23, n. 1, p. 39-41, Jan. 1992.

KATO, G.; NAKABAYASHI, N. The Durability of Adhesion to Phosphoric Acid Etched, Wet Dentin Substrates. Dent. Mater., Dallas, v.14, n.5, p. 347-352, Sept. 1998.

KONNO, A. N. K. et al. Storage Effect on the Shear Bond Strength of Adhesive Systems. Braz. Dent. J., Ribeirão Preto, v.14. n.1, p.42-47, 2003.

LELOUP, G. et al. Meta-analytical Review of Factors Involved in Dentin Adherence. J. Dent. Res., Washington, v.80, n.7, p. 1605-1614, July 2001.

LODOVICI, E. et al. Influência do Tempo de Armazenamento na Resistência de União à Dentina de Dois Tipos de Adesivos Dentários por Ensaio de Microtração. Ci. Odontol. Bras., São José dos Campos, v.6, n.4, p. 79-86, out./dez. 2003.

MATOS, A. B. et al. Estudo de Resistência à Tração de Três Sistemas Adesivos Associados à Resina Composta em Superfícies dentinárias. Pesq. Odontol. Bras., São Paulo, v.15, n.2, p. 161-165, abr./ jun. 2001.

MAURO, S. J. et al. Estudo da Resistência de União ao Cisalhamento de Diferentes Sistemas Adesivos à Dentina. Rev. Bras. Odontol., Rio de Janeiro, v. 57, n.4, p.222-226, jul./ago. 2000.

NAKABAYASHI, N.; KOJIMA, K.; MASUHARA, E. The promotion of adhesion by the infiltration of monomers into tooth substrates. J. Biomed. Mat. Res., Hoboken, v.16, n.3, p. 265-273, May 1982.

PASHLEY, D. H. et al. Collagen Degradation by Host-derived Enzymes During Aging. J. Dent. Res., Washington, v.83, n.3, p. 216-221, Mar. 2004. 
PERDIGÃO, J. RITTER, A. V. Adesão dos Tecidos Dentários. In: BARATIERI, L. N. et al. Odontologia Restauradora: Fundamentos e Possibilidades. São Paulo: Liv. Santos, p. 85-128, 2001.

REIS, A. et al. Sistemas Adesivos Atuais. J. Bras. Clin. Odontol. Integr., Curitiba, v. 5, n. 30, p. 455-466, dez. 2001/jan. 2002.

SADECK, F. T.; TAVARES, A. U.; CARDOSO, P. E. C. Efeito do Armazenamento de Adesivos de Frasco Único sobre a Resistência de União à Dentina. Ci. Odontol. Bras., São José dos Campos, v.6, n.2, p. 29-34, abr./jun. 2003.

SANO, H. et al. Nanoleakage: Leakage within the Hybrid Layer. Oper. Dent., Seattle, v. 20, n.1, p. 18-25, Jan./Feb. 1995.

Microtensile Testing, Nanoleakage and Biodegradation of Resin-dentin Bonds. J. Dent. Res., Washington, v.85, n.1, p. 11-14, Jan. 2006.

SANTOS, S. P.; PEREIRA, G. M.; ABDO, R. C. C. Avaliação da Resistência ao Cisalhamento da União Resina Composta/Porcelana em Função de Diferentes Tipos de Adesivos dentinários. J. Bras. Clin. Odontol. Integr., Curitiba, v. 4, n. 24, p. 35-38, nov. /dez. 2000.

SHIMADA, Y. et al. Shear Bond Strenght of Current Adhesive Systems to Enamel, Dentin and Dentin - enamel Junction Region. Oper. Dent., Seattle, v. 28, n. 5 p. 585-590, Sept./Oct. 2003.

; KIKUSHIMA, D.; TAGAMI, J. Micro-shear Bond Stregth of Resin-bonding Systems to Cervical Enamel. Am. J. Dent., San Antonio, v. 15, n. 6, p. 373-377, Dec. 2002.

SUSIN, AH. et al. Resistência de União à Tração de Sistemas Adesivos Atuais: Avaliação em Dentina e Esmalte. Rev. Odonto Ci., Porto Alegre, v. 19, n. 44, p. 145-151, abr./jun. 2004.

TAY, F. R.; PASHLEY, D. H. Water Treeing: a Potencial Mechanism for Degradation of Dentin Adhesives. Am. J. Dent., San Antonio, v. 16, n. 1, p. 06-12, Feb. 2003.

TAVARES, J. C.; CONCEIÇÃO, E. N. Resistência a Microtração de Três Sistemas Adesivos à Dentina. J. Bras. Clin. Odontol. Integr., Curitiba, v. 8, n. 44, p. 153-156, mar./abr. 2004.

VAN MEERBEEK, B. et al. Adhesion to Enamel and Dentin: Current Status and Future Challenges. Oper. Dent., Seattle, v. 28, n. 3, p. 215-235, May/June 2003. [Buonocore Memorial Lecture.]

WANG, H.; SHIMADA, Y.; TAGAMI, J. Shear Bbond Stability of Current Adhesive Systems to Enamel. Oper. Dent., Seattle, v. 29, n. 2 , p. 168-175, Mar./Apr. 2004. 